\section{Fístula arteriovenosa hepática em cão: relato de caso}

\author{
Hepatic arteriovenus fistula in dog - case report
} Letícia de Paula Siqueira Isidoro', Priscila Cardim de Oliveira², Taciana Oliveira Lopes', Mario Santos Filho ${ }^{3}$,
Cristiano Chaves Pessoa da Veiga ${ }^{4}$, Jonimar Pereira Paiva ${ }^{5}$ \& Julio Israel Fernandes ${ }^{5 *}$

'Médica veterinária. Programa de Residência em Área Profissional da Saúde, Universidade Federal Rural do Rio de Janeiro UFRRJ, Seropédica, RJ, Brasil

2Médica veterinária, Doutoranda. Programa de Pós-graduação em Ciências Veterinárias, Universidade Federal Rural do Rio de Janeiro - UFRRJ, Seropédica, RJ, Brasil

${ }^{3}$ Médico veterinário, Mestrando. Programa de Pós-graduação em Medicina Veterinária, Universidade Federal Rural do Rio de Janeiro - UFRRJ, Seropédica, RJ, Brasil

${ }^{4}$ Médico veterinário, Dr. Hospital Veterinário, Universidade Federal Rural do Rio de Janeiro - UFRRJ, Seropédica, RJ, Brasil

${ }^{5}$ Médico veterinário, Dr. Departamento de Medicina e Cirurgia Veterinária, Universidade Federal Rural do Rio de Janeiro UFRRJ, Seropédica, RJ, Brasil

\section{Resumo}

Fístulas arteriovenosas hepáticas são comunicações vasculares entre a artéria hepática e a veia porta fazendo com que o sangue se desvie da rede sinusóide hepática e retorne por fluxo retrógrado para o sistema portal. Essa afecção de rara ocorrência, pode ser de caráter congênito ou adquirido, podendo levar à anorexia, perda de peso, diarreia, alterações neurológicas e ascite, e possui o desvio portossistêmico como principal diagnóstico diferencial. O tratamento clínico é paliativo, sendo necessária a realização de cirurgia para resolução definitiva da doença. O presente relato reporta um caso de fistula arteriovenosa em cão, desde o início dos sinais clínicos, o diagnóstico e tratamento exclusivamente clínico realizado, tendo o animal uma sobrevida de 19 meses.

Palavras-chave: vascularização hepática, canino, tratamento.

\begin{abstract}
Hepatic arteriovenous fistulas are vascular communications between a hepatic artery and a portal vein causing the blood to bypass the hepatic sinusoid network and return by retrograde flow to the portal system. This rare occurrence can be congenital or acquired, and can lead to anorexia, weight loss, diarrhea, neurological and ascites, and has portosystemic deviation as the main diagnosis. The clinical treatment is palliative, requiring surgery to definitively resolve the disease. The present report reports a case of arteriovenous fistula in dogs, from the beginning of the clinical signs, the diagnosis and exclusively clinical treatment carried out, having a survival approximately 19 months.
\end{abstract}

Keywords: Hepatic vascularization, canine, treatment.

\section{Introdução}

As fístulas arteriovenosas intra-hepática (FAVH), são comunicações vasculares entre a artéria hepática e a veia porta fazendo com que o sangue se desvie da rede sinusóide hepática e retorne por fluxo retrógrado para o sistema portal. Essas afecções podem afetar um ou mais lobos hepáticos, sendo o lobo medial direito o mais acometido (Johnson, 2004).

De rara ocorrência, as FAVH podem ter causas congênitas, traumáticas ou neoplásicas (Johnson, 2004). Em cães e gatos a maioria das FAVH são congênitas, provavelmente refletindo a falha na diferenciação das estruturas venosas e arteriais embrionárias (Hopper et al., 1983).

As principais causas de FAVH adquirida incluem trauma abdominal, cirurgias hepáticas, neoplasias hepáticas, ruptura de aneurisma, obstrução da veia hepática e cirrose. As FAVH são diagnosticadas com mais frequência em cães com idade inferior a um ano e meio de idade, sendo a média de seis meses de idade (Johnson, 2004).

As manifestações clínicas de animais com FAVH são: anorexia, perda de peso, diarreia, êmese, dor abdominal, falha de crescimento, poliúria, polidipsia, febre, alteração neurológica e ascite. As alterações neurológicas estão relacionadas com a encefalopatia hepática (Chanoit et al., 2007).

\section{B] $\mathrm{M}$ \\ Brazilian Journal of Veterinary Medicine}

p-ISSN 0100-2430

Como citar: Isidoro, L. P. S., Oliveira, P. C., Lopes, T. O., Santos Filho, M., Veiga, C. C. P., Paiva, J. P., \& Fernandes, J. I. (2017). Fístula arteriovenosa hepática em cão: relato de caso. Brazilian Journal of Veterinary Medicine, 39(2), 120-125. doi: 10.29374/2527-2179.bjvm088917

Fonte de financiamento: Os exames foram realizados com recursos finaceiros do tutor e os custos de publicação serão financiados pelos autores

Conflito de interesses: Os autores declaram não haver conflito de interesses que precisam ser informados.

Recebido: Setembro 14, 2016.

Aceito: Maio 16, 2017.

Oestudo foi realizado no Hospital Veterinário de Pequenos Animais, Universidade Federal Rural do Rio de Janeiro - UFFRJ, Seropédica, RJ, Brasil.

\section{*Correspondência \\ Julio Israel Fernandes}

Programa de Pós-graduação em Medicina Veterinária, Universidade Federal Rural do Rio de Janeiro - UFRRJ

$\mathrm{Br} 465, \mathrm{Km} 7$

CEP 23897-000 - Seropédica (RJ), Brasil

E-mail: vetjulio@yahoo.com.br

Copyright Isidoro et al. Este é um artigo publicado em acesso aberto (Open Access) sob a licença Creative Commons Attribution Non-Commercial, que permite uso, distribuição e reproduçãa em qualquer meio, sem restrições desde que sem fins comerciais e que o trabalho original seja corretamente citado. 
Os animais com essa enfermidade podem apresentar sinais de insuficiência cardíaca, devido ao aumento do débito cardíaco (Hoskins, 1997).

Para a confirmação do diagnóstico é necessário a realização de exames laboratoriais e de imagem. A alteração hematológica mais comum é a anemia microcítica normocrômica não regenerativa. Não se sabe a causa da anemia microcítica, estudos sugerem uma deficiência no transporte de ferro, diminuição da concentração de ferro e aumento do depósito de ferro nas células de Kupffer. No hemograma, pode-se encontrar leucocitose, que pode estar associado com escape de endotoxinas ou bactérias do sistema porta por DPS (Berent \& Tobias, 2009; Mankin, 2015).

As anormalidades mais comuns no exame bioquímico são: hipoalbuminemia, diminuição do nitrogênio ureico sanguíneo (BUN), hipocolesterolemia, hipoglicemia, um aumento de 2 a 3 vezes de Fosfatase alcalina (FA) e Asparato amino transferase (AST) (Johnson, 2004).

Densidade urinária diminuída, presença de cristais de biurato de amônia e proteinúria podem ser observadas nos animais. Acredita-se que a baixa densidade urinária ocorra devido à polidpsia e baixo gradiente de concentração medular. A formação dos cristais de biurato de amônio ocorre devido ao metabolismo inadequado do ácido úrico que leva uma excreção excessiva de urato no rim e pela hiperamonúria que se forma a partir da deficiência do ciclo da ureia. Já a proteinúria acontece secundária à esclerose glomerular ou outra glomerulopatia. Animais com FAVH podem apresentar ascite, cujo o líquido drenado é classificado como transudato puro (Berent \& Tobias, 2009).

A ultrassonografia abdominal é o método mais simples e, muitas das vezes, o diagnóstico para a fístula arteriovenosa hepática. Os principais achados no exame ultrassonográfico são: ascite, presença de desvios portossistêmicos extra-hepáticos, redução do tamanho do fígado, modificações na arquitetura renal, artéria celíaca alargada, veia hepática alargada e a presença de cálculo na vesícula biliar (Szatmári et al., 2000). A utilização do Doppler é importante, pois animais que apresentam FAVH possuem anormalidades no fluxo do ramo da veia portal. Isso faz com que ocorra um retorno do sangue por fluxo retrogrado no sistema portal (Szatmári et al., 2000; Chanoit et al., 2007; Berent \& Tobias, 2009).

A angiografia por tomografia computadorizada (angioTC), realizada por administração periférica de contraste a base de iodo, permite a visualização da vascularização hepática incluindo artérias, veias e veias porta permitindo uma completa avaliação. A angioTC ainda apresenta como vantagens a capacidade para diagnosticar anomalias vasculares e a observação de alterações hemodinâmicas. Esteéum método seguro e minimamente invasivo para o diagnóstico de FAVH (Zwingenberger et al., 2005).

O principal diagnóstico diferencial da FAVH é o DPS pelo fato de ter as mesmas características clínicas (Johnson, 2004). Além do DPS, incluem como diagnósticos diferenciais: fibrose hepática, hipoplasia da veia porta, hepatite lobular dissecante, hipertensão portal não-cirrótica idiopática, defeitos cardíacos congênitos que causam insuficiência cardíaca congestiva direita e hipoproteinemia (Johnson, 2004).

O tratamento definitivo descrito para FAVH é cirúrgico, porém, antes da realização deste procedimento, se faz necessário o tratamento clínico. A terapêutica constitui em tratar a encefalopatia hepática (EH), a ascite, a hipertensão portal e a fibrose hepática (Chanoit et al., 2007).

De acordo com as principais bases de dados nacionais consultadas, esteé o primeiro relato de fistula arteriovenosa hepática descrito na literatura nacional, sendo também o primeiro relato com tratamento exclusivamente clínico, com sobrevida de 19 meses.

\section{Histórico}

Foi atendido no hospital veterinário da Universidade Federal Rural do Rio de Janeiro (HV-UFRRJ), um canino, sem raça definida, fêmea, de cinco meses de idade, com o histórico de ascite, hiporexia, emagrecimento, diarreia, mudança de comportamento e desorientação. Ao exame físico apresentou: temperatura $38,9^{\circ} \mathrm{C}$, frequência cardíaca 100 bpm, frequência respiratória $40 \mathrm{mpm}$, linfonodos mandibulares aumentados e abdômen distendido por ascite.

Frente ao quadro, foram solicitados exames laboratoriais e de imagem. As alterações observadas no hemograma foram: anemia microcítica normocrômica (hematócrito: 28,4\%) leucocitose $(27.400 \mu \mathrm{L})$, neutrofilia $(20.824 \mu \mathrm{L})$, monocitose $(3014 \mu \mathrm{L})$, presença de linfócito reativos e anisocitose. Os resultados da bioquímica sérica foram: hipoproteinemia (4,3g/dL), hipoalbuminemia (2,0g/dL), 
hipoglobulinemia (2,3g/dL), aumento da AST (52U/L) e FA (184U/L), GGT (5,0 U/L), ALT (72 U/L), bilirrubina total (0,3 mg/dL) e bilirrubina direta (0,1 mg/dL) normais. Na urinálise constatou-se: urina com cor palha e aspecto turvo, proteinúria, hematúria e presença de cristais de urato amorfo.

Com o objetivo de elucidar a causa da distensão abdominal solicitou-se ultrassonografia abdominal total, que permitiu evidenciar em modo B: efusão peritoneal com baixa celularidade, lobo hepático esquerdo pequeno com contornos irregulares e grande distensão vascular adjacente ao lobo hepático esquerdo com inúmeros vasos distendidos e tortuosos adjacentes (Figura 1). A anomalia vascular do lobo hepático esquerdo ao modo Doppler colorido direcional apresentava características de turbilhonamento sanguíneo, e na avaliação da onda espectral foi observado sinais acima e abaixo da linha base com características arteriais (pico sistólico) e venosas (fluxo laminar e contínuo). Foi utilizado aparelho ultrassonográfico Samsung Medson Sonoace r3 sonda microconvexa 4 a 7 MHz. (Figura 2).

Frente aos resultados dos exames laboratoriais e da ultrassonografia suspeitou-se de FAVH. Assim, foi solicitado a AngioTC para confirmação deste diagnóstico. Foi instituído tratamento clínico com ranitidina $2 \mathrm{mg} / \mathrm{kg}$ BID, metronidazol $15 \mathrm{mg} / \mathrm{kg}$ BID, furosemida $2 \mathrm{mg} / \mathrm{kg}$ SID, lactulose 0,5 ml/kg BID e alimentação (ração comercial) com baixa teor de proteína.

O retorno ocorreu em sete dias e o tutor relatou diminuição do volume abdominal, ausência da desorientação, melhora no comportamento e fezes normais.

Foi realizada tomografia computadorizada de abdômen com aquisição helicoidal com cortes transversais de $2 \mathrm{~mm}$, antes e após a injeção do meio de contraste iodado não iônico (iohexol $331 \mathrm{mgI} / \mathrm{kg}$ ) por via intra-venosa e observado: Fígado com dimensões acentuadamente diminuídas bordas regulares, atenuação e captação diminuídas, apresentando múltiplos vasos tortuosos de até $1,4 \mathrm{~cm}$, comunicantes ao vaso portohepático esquerdo e sugerindo componente de ramo da artéria hepática, sem comunicação sistêmica evidente; Aumento de calibre de veia porta e

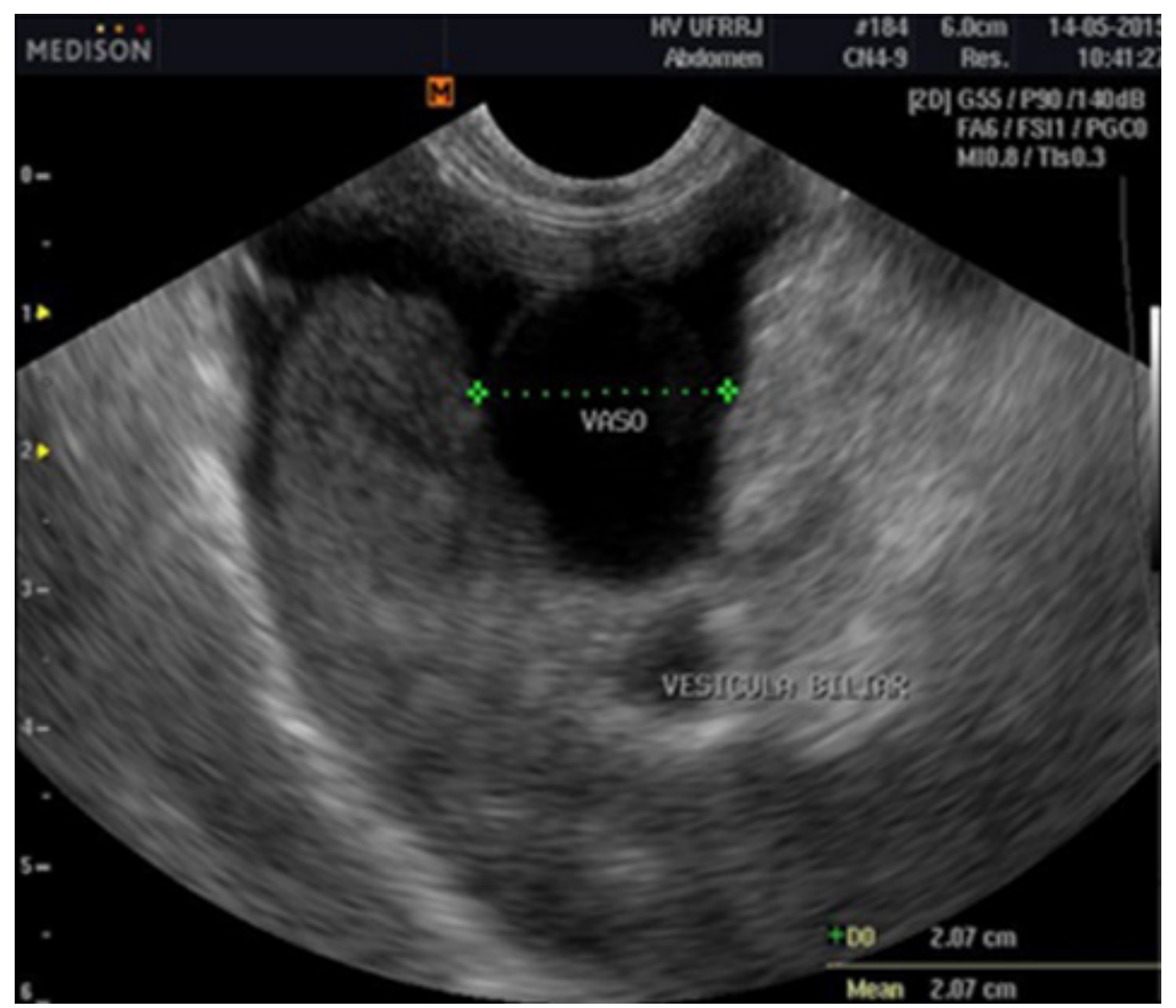

Figura 1. Imagem ultrassonográfica do fígado em modo B. Nota-se grande distensão vascular. 
moderada neovascularização mesentérica; Vesícula biliar de dimensões normais, com conteúdo denso de fundo (lama biliar); Pâncreas de volume levemente aumentado, com morfologia e contornos normais, impregnando-se homogeneamente, sem alterações significativas; Baço com dimensões normais, topografia e densidade usuais, com bordos regulares e captação homogênea; Rins em topografia usual, de volume, densidade e contorno normais, concentrando e excretando o meio de contraste, sem distensão de pelves ou ureteres; cálculo hiperdenso em pelve renal esquerda com cerca 0,7 x 0,5 x 0,5cm; Adrenais sem alterações significativas; Vesícula urinária apresentando parede lisas e integras, com deslocamento à esquerda.

No exame de tomografia computadorizada foi diagnosticado como Hepatopatia crônica severa com imagem de Fístula arterio-venosa hepato-portal, hipertensão portal e ascite. Não foi evidenciado desvio porto-sistêmico (Figura 3).

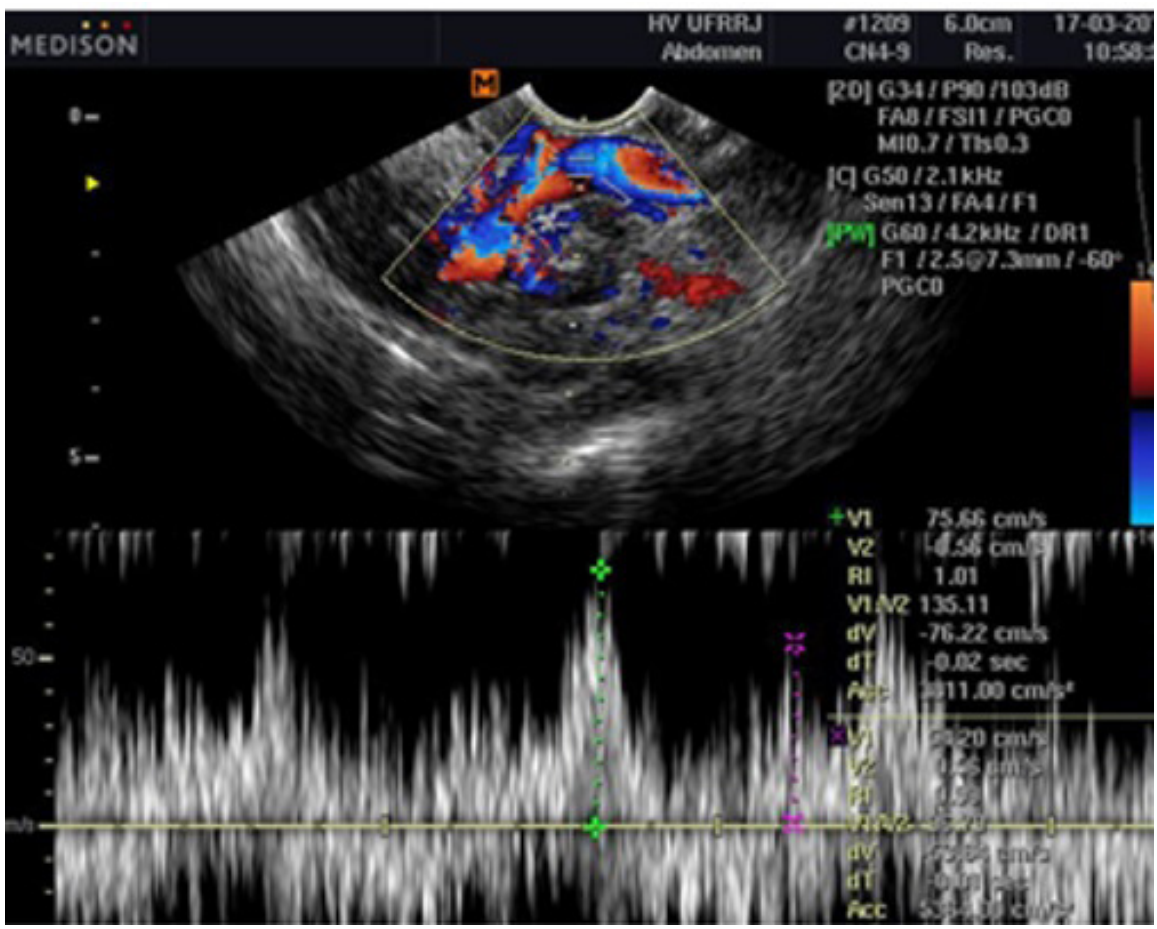

Figura 2. Imagem ultrassonográfica com Doppler colorido direcional e onda espectral da anomalia vascular do lobo hepático esquerdo. A presença de diferentes cores do mosaico (azul e vermelho) e sinais acima e abaixo da linha base na avaliação da onda espectral associadas as características arteriais (pico sistólico) e venosas (fluxo laminar e contínuo), são indicativas de FAVH.
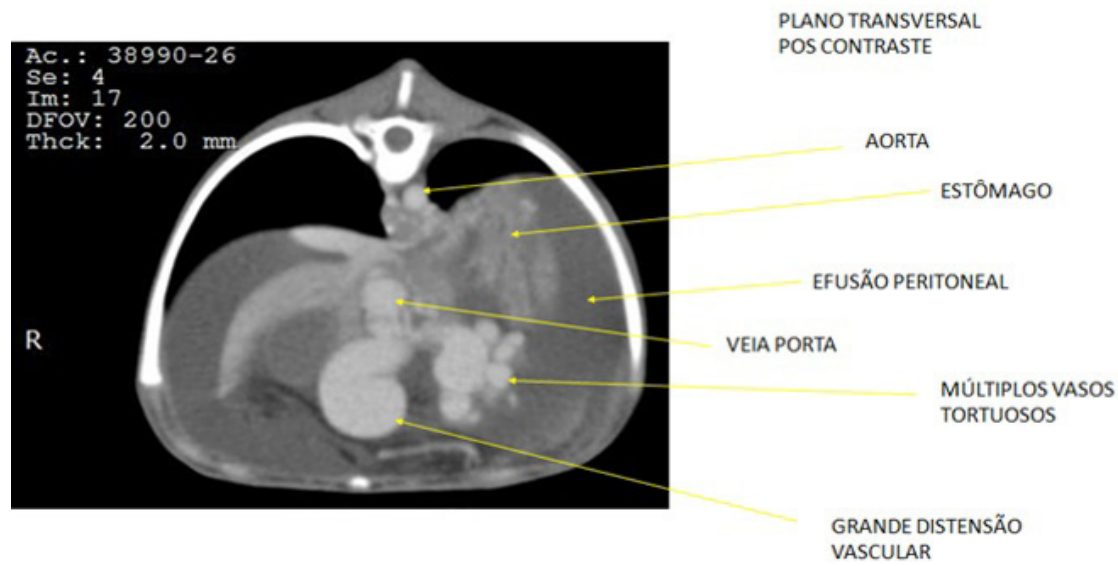

Figura 3. Imagem da tomografia contrastada feita no plano transversal, que indica a presença de hipertensão portal, efusão pleural e fístula arteriovenosa hepática. 
Após a confirmação do diagnóstico de FAVH instituiu-se tratamento com albumina (1 colher de chá), espironolactona $2 \mathrm{mg} / \mathrm{kg}$ SID, lactulose 0,5 ml/kg BID, enalapril 0,25 mg/kg BID e ração com baixa teor de proteína. Após dois meses de tratamento o animal estava clinicamente bem e não apresentava ascite ou sinais de EH. Foi mantido apenas a dieta comercial com baixo teor de proteína. O procedimento cirúrgico foi colocado como possibilidade de correção definitiva, entretanto o tutor preferiu apenas a terapêutica clínica.

Um ano após o diagnóstico o animal não apresentou ascite ou sinais de EH. Foram realizados exames de acompanhamento, as alterações laboratoriais observadas foram anemia microcítica hipocrômica, hematócrito 35\%, hipoalbuminemia (2,1g/dL) discreta e diminuição da ureia (9,0mg/dL). Na ultrassonografia observou-se um urólito no rim esquerdo.

Com um ano e meio de tratamento a única alteração do exame clínico foram pigmentos na córnea decorrentes de depósito de cálcio. Os exames laboratoriais apresentam anemia microcítica normocrômica hematócrito (36,9\%), diminuição da ureia (10mg/dl), do colesterol (94mg/dl), dos triglicerídeos $(29 \mathrm{mg} / \mathrm{dl})$ e hipoalbuminemia $(2, \mathrm{Og} / \mathrm{dl})$ além de aumento discreto na ALT (151U/L) e AST (56U/L). O exame ultrassonográfico se manteve como o anterior. Foi prescrito novamente a albumina e iniciado silimarina $25 \mathrm{mg} / \mathrm{kg} / \mathrm{BID}$ e mantida a ração com baixo teor de proteína. O animal veio a óbito em outubro de 2016, 19 meses após o diagnóstico, em casa e segundo informações do tutor o animal apresentou apatia e palidez da mucosa oral vindo a óbito no mesmo dia no final da tarde.

\section{Discussão}

A FAVH congênita é uma doença rara e normalmente acomete animas jovens com menos de seis meses de vida de acordo com Johnson (2004). O relato descreve o caso de um canino, fêmea, sem raça definida de cinco meses de idade.

De acordo com Berent \& Tobias (2009) e Mankin (2015), a anemia é comum nestes casos e a paciente apresentou esta alteração em todos os exames podendo estar relacionada com deficiência no transporte de ferro, diminuição da concentração deste ou aumento do depósito deste elemento nas células de Kupffer. Leucocitose pode ocorrer secundário ao estresse, inadequada liberação de endotoxinas hepáticas e bacterianas (Tobias \& Besser, 1997). As alterações bioquímicas corroboram com Berent \& Tobias (2009), sendo comum encontrar hipoalbuminemia, hipoglobulinemia e as enzima hepáticas séricas (FA, ALT e AST), estarem dentro da faixa de normalidade ou apresentarem discreto aumento.

No primeiro exame o animal apresentou hipoalbuminemia e aumento de FA e AST. No último exame apresentou hipoproteinemia, diminuição de ureia, colesterol e triglicerídeo e aumento ALT e AST. A hipocolesterolemia e diminuição do nitrogênio ureico sanguíneo (BUN) foi relatada por Johnson, 2004. As anormalidades encontradas no exame de urina, cor palha e aspecto turvo, presença de proteína, hemácias e cristais de urato amorfo também já foram descritas por Berent \& Tobias (2009). Amonemia decorrente de deficiências no ciclo da amônia associada ao metabolismo inapropriado do ácido úrico resultam maior excreção renal e assim formação dos cristais (Winkler et al., 2003).

As alterações encontradas na ultrassonografia como distensão vascular e o turbilhonamento observado pelo Dopller em modo B, corroboram com as reportadas por Szatmári et al. (2000) e Berent \& Tobias (2009). A angio TC é um método seguro e eficaz para o diagnóstico da FAVH de acordo com Zwingenberger et al. (2005) e pôde ser observado distensão e tortuosidade dos vasos o que nos possibilitou estabelecer o diagnóstico de FAVH.

Na medicina humana, uma maneira de reduzir a hipertensão portal é utilizando a administração de inibidores da enzima conversora da angiotensina (ECA), pois eles diminuem o gradiente de pressão venosa hepática portal (Tandon et al., 2010). Nesse trabalho foi utilizado o maleato de enalapril na tentativa de diminuir a hipertensão portal e os seus efeitos. Foi observado que a efusão peritonial cessou quando foi associado ao diurético oral o inibidor da ECA.

A suplementação de albumina em pacientes com hipoalbuminemia é algo controverso tanto na medicina humana quanto na medicina veterinária. Em animais com hipoalbuminemia e aumento da permeabilidade capilar, a suplementação de albumina frequentemente leva ao maior extravasamento desta através da membrana capilar (Uhing, 2004).

Na medicina humana o uso da suplementação de albumina para correção da hipoalbuminemia em doentes críticos não está recomendado, uma vez que não mostra benefícios clínicos. Porém, em pacientes com ascite sob tensão ou refratária, maior que cinco litros, o uso de albumina é considerado (Trabulo et al., 2012). O paciente foi suplementado com albumina e os níveis séricos 
aumentaram quando todas as medidas em conjunto foram adotadas, o que corrobora com Uhing (2004) que reportou a normalização da albumina à medida que foi tratada a causa inicial da hipoalbuminemia.

O tratamento clínico dessa afecção na literatura é indicado como estabilização do estado de saúde para indicar, em momento oportuno, o procedimento cirúrgico. Porém neste relato, a tutora optou por não realizar a cirurgia, sendo mantido apenas o tratamento clinico, obtendo-se boa qualidade de vida para o paciente e controle da encefalopatia e ascite, que são as complicações mais graves desta doença e que, de fato, não ocorreram no animal acompanhado durante 19 meses após o diagnóstico.

De acordo com Chanoit et al. (2007), cães tratados cirurgicamente com as técnicas de embolização com cola e hepatectomia tiveram sobrevida de 15 a 24 meses e todos necessitaram de algum tratamento clinico medicamentoso e/ou dieta. Não foram encontrados trabalhos que relatem o prognóstico e a evolução de um animal com fístula arteriovenosa hepática mantido apenas com tratamento clínico o que torna o relato inédito. Desta forma, não é possível extrapolar possíveis complicações decorrentes do tratamento exclusivamente clínico e a expectativa de vida destes animais.

\section{Conclusão}

O tratamento clínico instituído, foi eficaz no controle dos sintomas decorrentes da FAVH, garantindo qualidade de vida ao paciente e expectativa de vida próxima ao relato em pacientes que realizaram o tratamento cirúrgico.

\section{Referências}

Berent, A. C., \& Tobias, K. M. (2009). Portosystemic vascular anomalies. The Veterinary Clinics of North America. Small Animal Practice, 39(3), 513-541. http://dx.doi.org/10.1016/j.cvsm.2009.02.004. PMid:19524792.

Chanoit, G., Kyles, A. E., Weisse, C., \& Hardie, E. M. (2007). Surgical and interventional radiographic treatment of dogs with hepatic arteriovenous fistulae. Veterinary Surgery, 36(3), 199-209. http://dx.doi.org/10.1111/j.1532950X.2007.00263.x. PMid:17461943.

Hopper, P. E., Jongeward, S. J., \& Lammerding, J. J. (1983). Carotid body tumor associated with an arteriovenous fistula in a dog. The Compendium on Continuing Education for the Practicing Veterinarian, 5, 68-72.

Hoskins, J. D. (1997). Pediatria veterinária: cães e gatos do nascimento aos seis meses. 2.ed. Rio de Janeiro: Interlivros.

Johnson, S. E. (2004). Hepatopatias crônicas. In S. J Ettinger, \& E. C. Feldman (Eds.), Tratado de medicina interna veterinária (pp. 1369-1398). Rio de Janeiro: Guanabara Koogan.

Mankin, K. M. T. (2015). Current concepts in congenital portosystemic shunts. The Veterinary Clinics of North America. Small Animal Practice, 45(3), 477-487. http://dx.doi.org/10.1016/j.cvsm.2015.01.008. PMid:25737000.

Szatmári, V., Németh, T., Kótai, I., Vörös, K., \& Sótonyi, P. (2000). Doppler ultrassonographic diagnosis and anatomy of congenital intrahepatic arterioportal fistula in a puppy. Veterinary Radiology \& Ultrasound, 41(3), 284-286. http://dx.doi.org/10.1111/j.1740-8261.2000.tb01492.x. PMid:10850881.

Tandon, P., Abraldes, J. G., Berzigotti, A., Garcia-Pagan, J. C., \& Bosch, J. (2010). Renin-angiotensin-aldosterone inhibitors in the reduction of portal pressute: a systematic review and meta-analysis. Journal of Hepatology, 53(2), 273-282. http://dx.doi.org/10.1016/j.jhep.2010.03.013. PMid:20570385.

Tobias, K. M., \& Besser, T. E. (1997). Evaluation of leukocytosis, bacteremia, and portal vein partial oxygen tension in clinically normal dogs and dogs with portosystemic shunts. Journal of the American Veterinary Medical Association, 211(6), 715-718. PMid:9301740.

Trabulo, D., Cardoso, C., Mangualde, J., Freire, R., Cremers, I., \& Oliveira, A. P. (2012). Utilização e administração racional de albumina humana intra-hospitalar. GE Jornal Português de Gastrenterologia, 19(6), 300-307. http:// dx.doi.org/10.1016/j.jpg.2012.07.012.

Uhing, M. R. (2004). The albumin controversy. Clinics in Perinatology, 31(3), 475-488. http://dx.doi.org/10.1016/j. clp.2004.03.018. PMid:15325533.

Winkler, J. T., Bohling, M. W., Tillson, D. M., Wright, J. C., \& Ballagas, A. J. (2003). Portosystemic shunts: diagnosis, prognosis and treatment of 64 cases (1993-2001). Journal of the American Animal Hospital Association, 39(2), 169-185. http://dx.doi.org/10.5326/0390169. PMid:12617545.

Zwingenberger, A. L., McLear, R. C., \& Weisse, C. (2005). Diagnosis of arterioportal fistulae in four dogs using computed tomographic angiography. Veterinary Radiology \& Ultrasound, 46(6), 472-477. http://dx.doi. org/10.1111/j.1740-8261.2005.00086.x. PMid:16396262. 